\title{
Caractéristiques et prise en charge du syndrome Uvéite-Glaucome-Hyphéma
}

\section{Characteristics and management of Uveitis-Glaucoma-Hyphema syndrome}

\author{
M. Duchêne, C. Iscar, M. Muraine, J. Gueudry*
}

Service d'ophtalmologie-centre hospitalo-universitaire Charles-Nicolle, 22, boulevard Gambetta, 76031 Rouen, France

\section{MOTS CLÉS}

Uvéite ;

Glaucome ;

Hyphéma ;

Implant

intraoculaire ;

Syndrome UGH

\begin{abstract}
Résumé
Introduction. - Le syndrome Uvéite-Glaucome-Hyphéma (UGH) est causé par le frottement d'un implant intraoculaire sur les structures du segment antérieur. Les implants de chambre antérieure en étaient de grands pourvoyeurs. Le but de cette étude a été de caractériser l'évolution et les facteurs prédisposants du syndrome UGH à l'heure où l'implantation en chambre postérieure est la règle.

Patients et méthodes. - Il s'agissait d'une étude rétrospective monocentrique de 30 cas de syndromes UGH pris en charge entre janvier 2014 et septembre 2018. Les données de l'examen clinique initial, le type d'implant incriminé et la prise en charge ont été analysés.

Résultats. - Trente yeux de 28 patients ont été inclus. Les implants responsables étaient suturés à l'iris $(15 / 30,50 \%)$, placés dans le sac $(6 / 30,20 \%)$, suturés à la sclère $(4 / 30,13,3 \%)$, positionnés dans le sulcus $(3 / 30,10 \%)$ ou avaient une position « in and out » $(2 / 30,6,7 \%)$. La prise en charge initiale a été médicale (18 yeux) ou chirurgicale (12 yeux). Les procédures chirurgicales ont consisté en une explantation $(n=4)$, un repositionnement de l'implant $(n=7)$ ou une trabéculectomie $(n=1)$. Les récidives sont survenues avec le traitement médical $(9 / 18)$, sans récidive dans le groupe chirurgical $(p=0,02)$. L'hypertonie oculaire s'est chronicisée dans 19 cas sur $30(63,3 \%)$.
\end{abstract}




\section{KEYWORDS \\ Uveitis; \\ Glaucoma; \\ Hyphema; \\ Intraocular lens; \\ UGH syndrome}

\section{Introduction}

Le syndrome Uvéite-Glaucome-Hyphéma (UGH) est une complication rare, mais potentiellement sévère de la chirurgie de la cataracte. Initialement décrit par Ellingson et al., en 1978 pour les implants intraoculaires de première génération, ce syndrome apparaît lorsqu'il existe un contact de l'implant intraoculaire avec les structures du segment antérieur entraînant un frottement. Il est donc responsable d'une inflammation par rupture de la barrière hémato-aqueuse, d'épisodes d'hyphéma/Tyndall hématique récurrents par effraction vasculaire ainsi que d'une élévation de la pression intraoculaire par dispersion pigmentaire pouvant évoluer vers un glaucome [1]. L'incidence de ce syndrome a diminué par l'amélioration constante de la conception des implants intraoculaires ainsi que des techniques chirurgicales [2]. Notre étude a pour objectif de caractériser l'évolution clinique et les facteurs favorisants du syndrome UGH, ainsi que sa prise en charge, à l'heure où l'implantation en chambre postérieure est la règle.

\section{Matériels et méthodes}

Nous rapportons une étude monocentrique, rétrospective, menée au CHU de Rouen entre janvier 2014 et septembre
2018. Tous les patients du service présentant un tableau complet d'UGH (Tyndall hématique ou hyphéma, hypertonie oculaire et atrophie irienne sectorielle), quelle que soit la position de l'implant intraoculaire, ont été inclus. Les tableaux d'UGH incomplets avec un Tyndall hématique en chambre antérieure, une atrophie irienne localisée en regard d'une partie de l'implant et/ou la présence d'un contact vu à l'échographie de segment antérieur en haute fréquence faisant preuve du mécanisme de frottement ont également été inclus. Nous avons défini comme atteinte glaucomateuse, la présence d'un déficit campimétrique objectivé. Les patients avec un recul minimal de 6 mois ont été inclus.

Les données concernant les 28 patients ( 30 yeux) ont été collectées : l'âge et le sexe, la date de la chirurgie initiale de cataracte ainsi que le type d'implant posé et le temps écoulé entre la chirurgie et le premier épisode d'UGH. Nous avons également relevé ; initialement, puis à 1 mois et 6 mois, la meilleure acuité visuelle corrigée, la pression intraoculaire (PIO), la présence d'hyphéma/microhyphéma, d'hémorragie intra-vitréenne et le traitement mis en œuvre.

Les analyses statistiques ont été effectuées à l'aide du logiciel Excel ${ }^{\circledR}$. Nous avons utilisé le test de Wilcoxon pour les analyses statistiques paramétriques appariées avant/après traitement, le test de Mann-Whitney et de Kruskal-Wallis pour comparer les variables quantitatives et le test de Fisher pour comparer les variables qualitatives. 

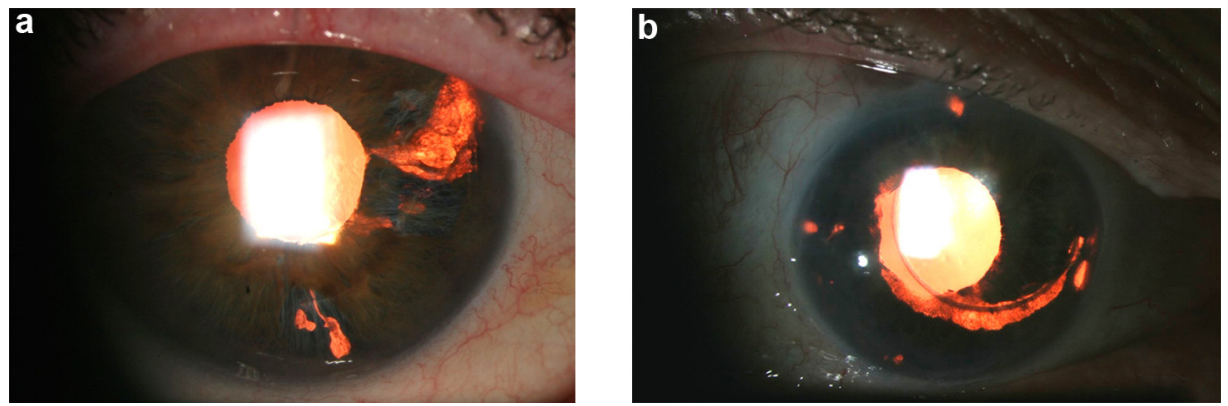

Figure 1. Syndrome UGH. Atrophie irienne sectorielle visible en rétro-illumination causée par le frottement localisé de l'haptique de l'implant positionné « in and out ». Noter la visualisation de l'haptique au travers de la zone d'atrophie (a). Atrophie irienne sectorielle visible en rétro-illumination causée par le frottement du bord de l'optique de l'implant suturé à l'iris (b).

\section{Résultats}

\section{Tableau clinique initial}

Trente yeux de 28 patients ont été inclus durant la période d'étude. Il y avait 6 femmes et 22 hommes. Les patients ont initialement consulté pour un flou visuel $(30 / 30,100 \%)$, une rougeur oculaire $(5 / 30,16,7 \%)$ et une douleur oculaire $(5 / 30,16,7 \%)$. À l'examen clinique initial étaient retrouvés un Tyndall hématique $(30 / 30,100 \%)$, un hyphéma $(4 / 30,13,3 \%)$, une hémorragie intra-vitréenne $(19 / 30$, $63,3 \%)$ et une atrophie irienne sectorielle $(22 / 30,73,3 \%)$ (Fig. 1 et 2). Ainsi, le syndrome UGH a été considéré comme complet $(20 / 30,66,7 \%)$ ou incomplet $(10 / 30$, $33,3 \%)$. L'atrophie irienne est survenue secondairement au cours du suivi (7 patients). L'âge moyen de survenue était de $65,9 \pm 14,7$ ans. Le délai entre l'implantation initiale et la survenue du syndrome UGH était de 32,5 mois $\pm 39,5$ mois.

\section{Type et positionnement des implants intraoculaires}

Dix-neuf yeux $(63,3 \%)$ étaient porteurs d'un implant trois pièces, 10 yeux $(33,3 \%)$ d'un implant monobloc ( 9 en acrylique et 1 en PMMA) et un œil n'était pas renseigné. Un implant fixé à l'iris était en cause chez 15 yeux sur $30(50 \%)$ dont 2 clippés et 13 suturés à sa face postérieure. Les autres implants en cause étaient positionnés dans le sac capsulaire $(6 / 30,20 \%)$, suturés à la sclère $(4 / 30,13,3 \%)$, dans le sulcus $(3 / 30,10 \%)$ et en position ectopique « in and out $»(2 / 30,6,7 \%)$. Dans les cas d'implants positionnés dans le sac, étaient retrouvés un iridodonésis/phakodonésis $(1 / 6)$, un implant positionné à l'envers (1/6), une antéro-position de l'implant par des reliquats cristalliniens $(1 / 6)$ et un décentrement tardif de l'implant (3/6). Une échographie du segment antérieur par une sonde haute fréquence a été effectuée devant l'absence d'atrophie irienne et la présence d'un implant dans le sac capsulaire ; ce qui a permis d'objectiver le contact entre l'implant et l'iris. Le premier épisode d'UGH survenait après l'implantation suturée à la sclère en moyenne à $10,25 \pm 29,7$ mois, suturée à l'iris à $22,21 \pm 24,1$ mois, dans le sulcus à $45 \pm 55,2$ mois et dans le sac à $84,5 \pm 58,7$ mois $(p=0,34)$.

\section{Prise en charge des patients}

La décision thérapeutique a été prise au cas par cas en fonction de l'histoire clinique. Dans la figure 3, la prise en charge thérapeutique en fonction de la position de l'implant est décrite (Fig. 3). Au final, 12 yeux ont été traités chirurgicalement d'emblée ( $40 \%$ ) et 5 yeux ayant récidivé dans le groupe médical secondairement, soit $56,7 \%$ de l'ensemble des cas d'UGH. La technique chirurgicale privilégiée quand cela était réalisable, était une fixation de l'implant à la sclère $(9 / 17)$, puis l'explantation $(5 / 17)$, puis le repositionnement de l'implant dans le sac $(2 / 17)$ et la fixation à l'iris $1(1 / 17)$. Une trabéculectomie puis un cycloaffaiblissement ont été effectués du fait d'une hypertonie majeure initiale persistante $(1 / 17)$. Dans le groupe de patients traités initialement médicalement, un traitement par corticoïdes locaux $(13 / 18$, $72,2 \%)$ et par hypotonisant en collyre ou systémique $(15 / 18$, $83,3 \%)$ ont été utilisés. Les patients traités médicalement étaient plus âgés que les patients traités chirurgicalement $(71,5 \pm 11,9$ ans contre $57,8 \pm 14,8$ ans, $p=0,007)$.

\section{Acuité visuelle}

La meilleure acuité visuelle corrigée a progressé significativement de 1,29 $\pm 0,81 \log$ MAR initialement à $0,53 \pm 0,62 \log$ MAR $(p<0,001)$ à 1 mois, puis à $0,64 \pm 0,78 \log$ MAR $(p=0,001)$ à 6 mois, sans différence significative entre le traitement initial médical ou chirurgical $(0,73 \pm 0,92 \log$ MAR versus $0,51 \pm 0,61 \log \operatorname{MAR}(p=0,82)$ à 6 mois de suivi).

\section{Pression intraoculaire}

La pression intraoculaire a significativement diminué de $31,12 \pm 11,36 \mathrm{mmHg}$ initialement à $19,0 \pm 6,94 \mathrm{mmHg}$ à 1 mois $(p<0,001)$ et à $15,6 \pm 4,02 \mathrm{mmHg}$ à 6 mois $(p<0,001)$. Un traitement hypotonisant au long cours a été mis en place pour 18 cas sur 30 (60\%). En moyenne, il fallait $2,16 \pm 0,92$ traitements hypotonisants.

Quatre cas sur 18 ont évolué vers une neuropathie glaucomateuse chez les patients ayant initialement bénéficié d'un traitement médical contre 3 sur 12 chez ceux opérés en première intention $(p=0,67)$. À distance du traitement initial, un traitement chirurgical de l'hypertonie a été nécessaire $(n=2)$. Un cas qui avait bénéficié d'une explantation et d'un cycloaffaiblissement a ensuite nécessité la pose d'un drain 

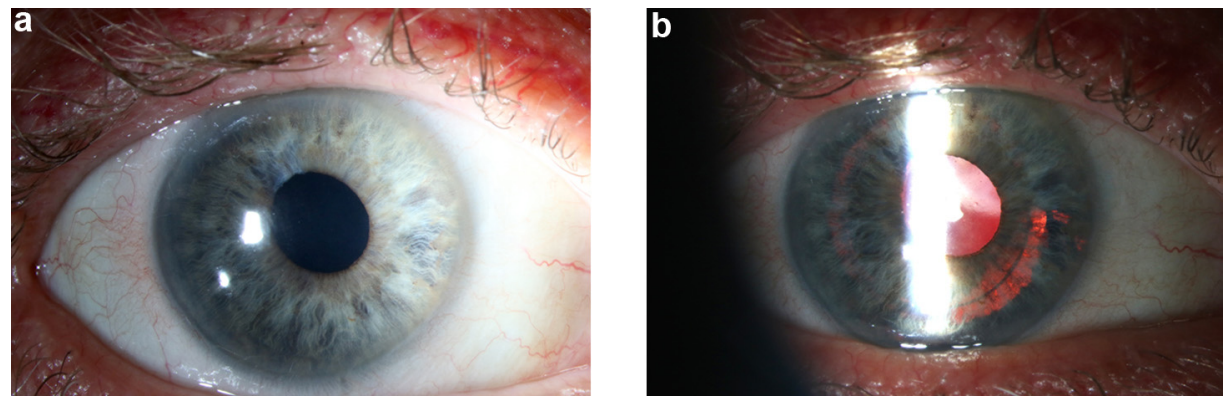

Figure 2. Syndrome UGH. Aspect à la lampe à fente avec le diffuseur objectivant un fin dépôt de pigment de l'iris en supérieur (a). L'examen en rétro-illumination démasque une atrophie irienne causée par le frottement du bord l'optique d'un implant intraoculaire positionné dans le sulcus (b).

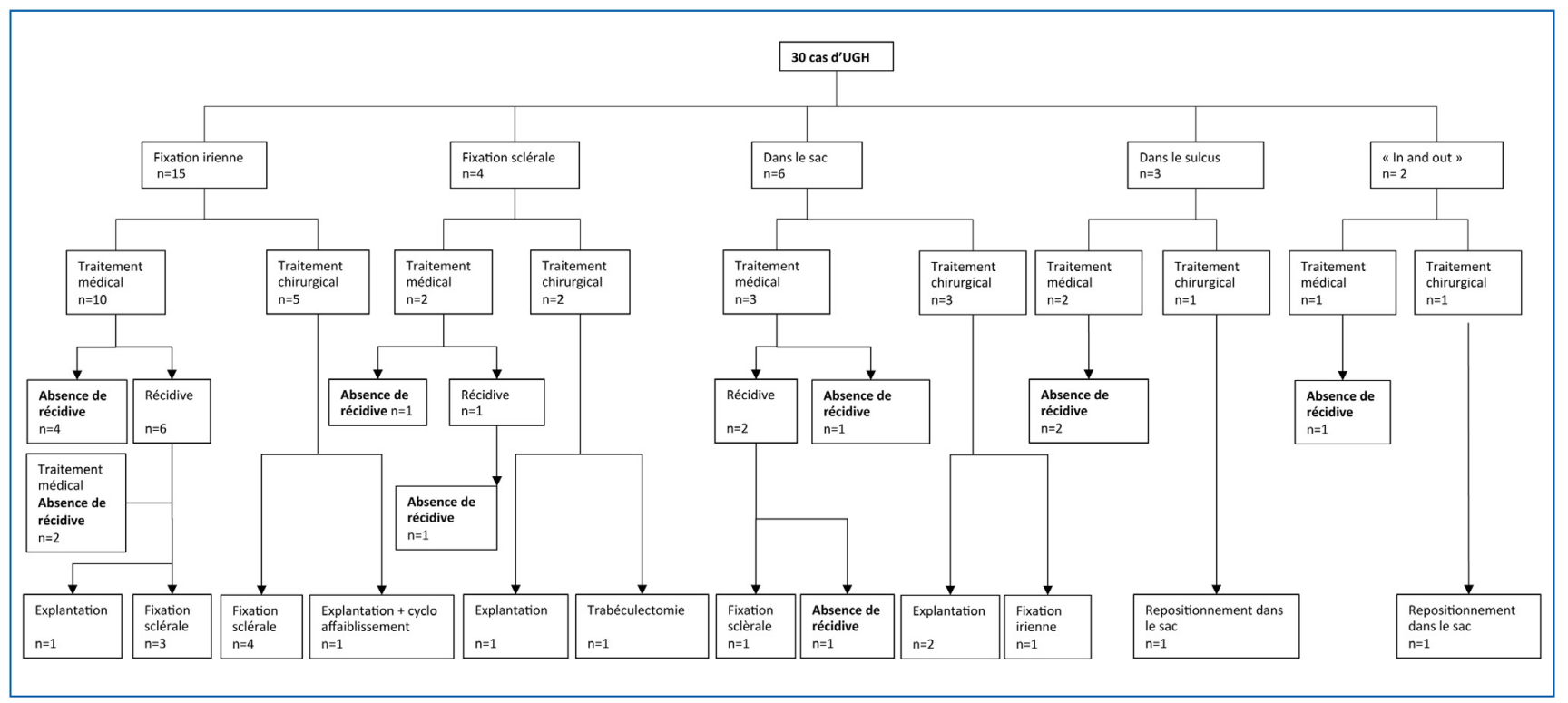

Figure 3. Prise en charge des patients présentant un syndrome Uvéite-Glaucome-Hyphema (UGH) en fonction du type d'implantation initiale. À noter qu'aucun patient traité chirurgicalement initialement ou après récidive n'a présenté de récidive d'UGH.

de Baerveldt devant l'apparition d'un glaucome réfractaire. Un cas initialement traité médicalement a bénéficié d'une trabéculectomie.

\section{Récidive du syndrome UGH}

Neuf récidives $(9 / 18,50 \%)$ ont été observées dans le groupe d'UGH traité médicalement initialement, en moyenne 8,3 mois $\pm 6,24$ après le premier épisode. Aucune récidive n'est survenue dans le groupe traité chirurgicalement en première intention avec un recul minimum de 6 mois $(p=0,02)$. Cinq cas traités initialement médicalement ont finalement bénéficié d'un traitement chirurgical (1 explantation et 4 implantations suturées à la sclère) dans le mois qui a suivi la récidive. Dans les 4 autres cas, le traitement médical a été efficace pour la prise en charge de la récidive.

\section{Discussion}

Le syndrome UGH a été initialement décrit avec les implants de chambre antérieure $[1,3,4]$. Ainsi, le frottement de l'haptique ou de l'optique de l'implant intraoculaire sur le tissu uvéal a pour conséquence l'apparition de signes antérieurs d'uvéite du fait de la rupture de la barrière hémato-aqueuse, d'hyphéma et d'une élévation de la pression intraoculaire par obstruction chronique trabéculaire associée à la dispersion pigmentaire [5,6]. Dans notre étude, les positions en dehors du sac capsulaire sont principalement représentées ; en premier lieu les implants suturés à l'iris (50\%), puis ceux positionnés dans le sulcus, puis ceux « in and out ». Ceci s'explique par la proximité de l'implant mettant les haptiques ou l'optique de l'implant directement en contact avec l'iris. Cependant, un implant positionné dans le sac capsulaire peut être également responsable d'UGH. Zhang et al. avancent dans ce cas de figure deux mécanismes: d'une part, un phakodonesis subclinique du complexe IOL- sac capsulaire avec une zonule lâche responsable du frottement et d'autre part, une fibrose de la capsule antérieure autour de l'haptique qui rentre au contact avec le tissu uvéal [7]. Des implants antéropositionnés, des implants décalés par contraction du sac $[8,9]$ ou par un syndrome de pseudo-exfoliation capsulaire [10], et la présence d'un kyste iridociliaire [11] ont été décrits dans 
la littérature et rendent le diagnostic plus difficile. Plusieurs implants positionnés dans le sac sont responsables d'un UGH dans notre série dont un cas d'implant antéro-positionné par des reliquats cristalliniens. Bryant et al. [12] avaient déjà proposé un mécanisme d'antéro-positionnement par un anneau de Sommerring pour expliquer un cas d'UGH. Le décentrement tardif du sac est une complication de la chirurgie de la cataracte et est la cause de nombreuses explantations d'implants ou de repositionnement [13-15]. Son incidence varie de 0,1 à $3 \%$ selon les études et il est responsable de 3 cas d'UGH dans notre étude.

Nous décrivons 4 syndromes UGH avec des implants suturés à la sclère pourtant loin des structures iriennes et uvéales. Ceci pourrait être expliqué par une configuration anatomique particulière à type de concavité de l'iris, qui pourrait provoquer tout de même un frottement implantiris alors que l'implant est positionné plus postérieurement que lorsqu'il se trouve dans le sac. Certains ont proposé qu'il pourrait exister un frottement des haptiques sur le tissu uvéal au niveau des volets scléraux, entraînant un hyphéma et une inflammation intraoculaire comme cela a été montré par Palvin et al. [16].

L'échographie par haute fréquence du segment antérieur peut mettre en évidence le contact entre l'implant et le tissu uvéal [17-20]. La tomographie par cohérence optique antérieure peut parfois mettre en évidence les frottements entre l'iris et l'implant comme cela a été montré par Werner [21].

L'explantation de l'implant intraoculaire est l'approche chirurgicale traditionnelle. Dans l'étude de Chan et al. [22], le syndrome d'UGH représente encore la troisième cause d'explantation, soit $10 \%$ des cas (9/98 explantations). Cependant, il semble nécessaire de proposer une alternative thérapeutique à l'explantation comme le changement de position d'implant afin de maintenir une bonne qualité visuelle. Jasinskas et al. [23] ont montré l'intérêt de la suture de l'implant à l'iris pour des syndromes UGH concernant des implants de sac. Cependant, la fixation de l'implant à l'iris est la plus grande pourvoyeuse de syndrome UGH dans notre étude. Il semble donc nécessaire de proposer un repositionnement de l'implant à la sclère afin de l'éloigner le plus possible des structures uvéales, même si cette intervention ne protège pas totalement d'éventuels frottements. De plus, la suture à l'iris semble donner plus de syndrome UGH que les implants clippés car le contact est plus marqué due à la configuration de ces implants.

Dans les cas où le traitement chirurgical doit être différé ou ne peut être effectué, un traitement médical conservateur, reposant principalement sur les corticoïdes collyres et des agents hypotonisants locaux, peut être proposé. Il ne faut pas négliger ce traitement car il a permis le contrôle de l'épisode d'UGH dans plus d'un tiers des cas dans notre étude. Une équipe a rapporté l'intérêt de l'iridoplastie par laser Argon [24]. D'autres équipes ont eu recours à la pose d'un anneau de tension capsulaire [7].

Quel que soit le traitement employé, il n'y a pas eu de différence significative concernant l'amélioration de l'acuité visuelle ou le contrôle tensionnel avec la limite du recul de cette l'étude. Une fois l'épisode aigu résolu, une récupération de l'acuité visuelle et une normalisation de la PIO, avec ou sans maintien d'un traitement hypotonisant, étaient observées. Aucune étude ne s'est intéressée à l'évolution vers une hypertonie oculaire chronique. Sept patients ont évolué vers une neuropathie glaucomateuse mais le recul actuel rend le jugement de l'apparition de cette maladie chronique difficile.

Les récidives sont survenues exclusivement chez des patients traités médicalement. On peut supposer que le traitement médical conservateur permet de contrôler l'inflammation aiguë, mais n'empêche pas la survenue de récidive car il ne supprime pas le frottement. Cependant, la survenue d'une atrophie irienne et d'une sclérose vasculaire au niveau de la zone de frottement peut faire que la récidive ne survienne pas malgré l'absence de traitement chirurgical. Il n'y a pas d'autres études ayant évalué le taux de récidives après traitement médical. En deuxième intention, une intervention chirurgicale a été réalisée chez plus de la moitié des patients ayant récidivé. Le traitement médical semble donc plutôt être à réserver à certains patients pour lesquels un traitement chirurgical semble trop risqué au niveau ophtalmologique ou au niveau général [25].

\section{Conclusion}

Le syndrome UGH syndrome est une complication rare de la chirurgie de la cataracte mais toujours d'actualité malgré l'évolution des techniques chirurgicales. Une prise en charge chirurgicale initiale permet d'éviter les récidives en supprimant le frottement de l'implant alors que la prise en charge médicale ne le permet pas toujours. Elle permet cependant de contrôler les poussées inflammatoires et tensionnelles lorsque le syndrome UGH survient sur des yeux fragiles. Quand cela est donc possible, il semble préférable de traiter chirurgicalement les syndromes UGH en déplaçant l'implant, en l'éloignant de l'iris le plus souvent ou plus rarement en le retirant.

\section{Financements}

Aucun.

\section{Déclaration de liens d'intérêts}

Les auteurs déclarent ne pas avoir de liens d'intérêts.

\section{Références}

[1] Ellingson FT. The uveitis-glaucoma-hyphema syndrome associated with the mark VIII anterior chamber lens implant. Am Intra-Ocul Implant Soc J 1978;4:50-3.

[2] Apple DJ, Mamalis N, Loftfield K, Googe JM, Novak LC, KavkaVan Norman D, et al. Complications of intraocular lenses. A historical and histopathological review. Surv Ophthalmol 1984;29:1-54.

[3] Hagan JC. A comparative study of the 912 and other anterior chamber intraocular lenses. Am Intra-Ocul Implant Soc J 1984;10:324-8.

[4] Khan YA, Pavlin CJ, Cykiert R, Rootman DS. Uveitis-glaucomahyphema syndrome after handmade, anterior chamber lens implantation. J Cataract Refract Surg 1997;23:1414-7. 
[5] Zemba M, Camburu G. Uveitis-Glaucoma-Hyphaema syndrome. General review. Romanian J Ophthalmol 2017;61:11-7.

[6] Mierlo CV, Pinto LA, Stalmans I. Surgical management of iatrogenic pigment dispersion glaucoma. J Curr Glaucoma Pract DVD 2015;9:28-32.

[7] Zhang L, Hood CT, Vrabec JP, Cullen AL, Parrish EA, Moroi SE. Mechanisms for in-the-bag uveitis-glaucoma-hyphema syndrome. J Cataract Refract Surg 2014;40:490-2.

[8] Boutboul S, Letaief I, Lalloum F, Puech M, Borderie V, Laroche L. Pigmentary glaucoma secondary to in-the-bag intraocular lens implantation. J Cataract Refract Surg 2008;34:1595-7.

[9] Badakere SV, Senthil S, Turaga K, Garg P. Uveitisglaucoma-hyphaema syndrome with in-the-bag placement of intraocular lens. BMJ Case Rep 2016;19, http://dx.doi.org/10.1136/bcr-2015-213745.

[10] Sousa DC, Leal I, Faria MY, Pinto LA. A rare manifestation of uveitis-glaucoma-hyphema syndrome. J Curr Glaucoma Pract 2016;10:76-8.

[11] Foroozan R, Tabas JG, Moster ML. Recurrent microhyphema despite intracapsular fixation of a posterior chamber intraocular lens. J Cataract Refract Surg 2003;29:1632-5.

[12] Bryant TK, Feinberg EE, Peeler CE. Uveitis-glaucoma-hyphema syndrome secondary to a Soemmerring ring. J Cataract Refract Surg 2017;43:985-7.

[13] Pueringer SL, Hodge DO, Erie JC. Risk of late intraocular lens dislocation after cataract surgery, 1980-2009: a populationbased study. Am J Ophthalmol 2011;152:618-23.

[14] Ford JR, Werner L, Owen L, Vasavada SA, Crandall A. Spontaneous bilateral anterior partial in-the-bag intraocular lens dislocation following routine annual eye examination. J Cataract Refract Surg 2014;40:1561-4.

[15] Fernández-Buenaga R, Alio JL, Pérez-Ardoy AL, LarrosaQuesada A, Pinilla-Cortés L, Barraquer R, et al. Late in-the-bag intraocular lens dislocation requiring explantation: risk factors and outcomes. Eye 2013;27:795-802.
[16] Pavlin CJ, Rootman D, Arshinoff S, Harasiewicz K, Foster FS. Determination of haptic position of transsclerally fixated posterior chamber intraocular lenses by ultrasound biomicroscopy. J Cataract Refract Surg 1993;19:573-7.

[17] Mostafavi D, Nagel D, Danias J. Haptic-induced postoperative complications. Evaluation using ultrasound biomicroscopy. Can J Ophthalmol 2013;48:478-81.

[18] Lima BR, Pichi F, Hayden BC, Lowder CY. Ultrasound biomicroscopy in chronic pseudophakic ocular inflammation associated with misplaced intraocular lens haptics. Am J Ophthalmol 2014;157:813-7.

[19] Piette S, Canlas OAQ, Tran HV, Ishikawa H, Liebmann JM, Ritch R. Ultrasound biomicroscopy in uveitis-glaucoma- hyphema syndrome. Am J Ophthalmol 2002;133:839-41.

[20] Wu N, Zhang H, Chen B, Ding W. A novel application of Bultrasonography at various head positions in the diagnosis of untypical uveitis-glaucoma-hyphema (UGH) syndrome: A case report. Medicine 2019;98:e13891.

[21] Werner L, Michelson J, Ollerton A, Leishman L, Bodnar Z. Anterior segment optical coherence tomography in the assessment of postoperative intraocular lens optic changes. J Cataract Refract Surg 2012;38:1077-85.

[22] Chan TCY, Lok JKH, Jhanji V, Wong VWY. Intraocular lens explantation in Chinese patients: different patterns and different responses. Int Ophthalmol 2015;35:679-84.

[23] Jasinskas V, Vaiciuliene R, Varoniukaite A, Speckauskas M. Novel microsurgical management of uveitis-glaucoma-hyphema syndrome. Int Ophthalmol 2019;39:1607-12.

[24] Walland MJ. Uveitis-glaucoma-hyphaema (UGH) syndrome treated with local laser iridoplasty: Letter to the editor. Clin Experiment Ophthalmol 2017;45:647-8.

[25] Kjeka O, Bohnstedt J, Meberg K, Seland JH. Implantation of scleral-fixated posterior chamber intraocular lenses in adults. Acta Ophthalmol (Copenh) 2008;86:537-42. 